\title{
CÁLCULO DA VARIAÇÃO DOS ELEMENTOS ORBITAIS DE SATÉLITES LUNARES DEVIDO À DISTRIBUIÇÃO NÃO UNIFORME DE MASSA DA LUA
}

\author{
Maria Lívia G. T. X. Da Costa ${ }^{1}$, Rodolpho Vilhena de Moraes ${ }^{1}$, Antônio F. B. A. Prado². \\ 1. Instituto de Ciência e Tecnologia, Universidade Federal de São Paulo-UNIFESP \\ 12231-289, São José dos Campos, SP, Brasil \\ E-mails: livia.thibes@gmail.com, rodolpho.vilhena@gmail.com
}

\author{
2. Instituto Nacional de Pesquisas Espaciais- INPE \\ 12227-010, São José dos Campos, SP, Brasil \\ E-mail: prado@dem.inpe.br
}

Resumo - Neste trabalho, utilizando as equações planetárias de Lagrange, são comparadas as variações de elementos orbitais de satélites lunares devido à distribuição não uniforme de massa da Lua com as variações de elementos orbitais de satélites artificiais terrestres devido à distribuição não uniforme de massa da Terra. Soluções analíticas aproximadas são comparadas com a integração numérica das equações para algumas simulações.

Palavras-chave - Astrodinâmica, satélites lunares, potencial gravitacional, elementos orbitais, satélites artificiais.

\section{Introdução}

Quando se estuda o potencial de um satélite artificial ao redor da Lua utilizando os polinômios de Legendre, observa-se que a ordem de grandeza de alguns coeficientes associados à ordem e grau dos polinômios não são hierarquicamente proporcionais à ordem e grau dos polinômios. Por exemplo, diferente do caso da Terra, a ordem do coeficiente associado ao $C_{22}$ é apenas um décimo menor que o coeficiente associado ao $J_{2}$; também, como exemplo para a Lua, a ordem de grandeza do coeficiente associado ao $J_{9}$ é maior que a ordem de grandeza do coeficiente associado ao $J_{3}$. Isto faz com que o comportamento do movimento orbital de satélites lunares, sob alguns aspectos, seja diferente do comportamento do movimento orbital de satélites artificiais da Terra. Na Tabela 1 são fornecidos alguns valores de tais coeficientes (Lemoine et al., 1998 e Chen et al., 2005).

Tabela 1. Valores de coeficientes harmônicos para a Terra e para a Lua.

\begin{tabular}{|c|c|c|}
\cline { 2 - 3 } \multicolumn{1}{c|}{} & Terra & Lua \\
\hline $\boldsymbol{C}_{20}=-\boldsymbol{J}_{2}$ & $1,082516.10^{-3}$ & $2,032337.10^{-4}$ \\
\hline $\boldsymbol{C}_{22}$ & $1,57443228.10^{-6}$ & $2,2357.10^{-5}$ \\
\hline $\boldsymbol{C}_{30}=-\boldsymbol{J}_{3}$ & $-2,532656026.10^{-6}$ & $8,47590.10^{-6}$ \\
\hline $\boldsymbol{C}_{40}=-\boldsymbol{J}_{4}$ & $-1,655470.10^{-6}$ & $-9,5919310.10^{-6}$ \\
\hline $\boldsymbol{C}_{50}=-\boldsymbol{J}_{5}$ & $-2,272959251.10^{-7}$ & $7,15409.10^{-7}$ \\
\hline $\boldsymbol{C}_{60}=-\boldsymbol{J}_{\boldsymbol{6}}$ & $5,406524138.10^{-7}$ & $-2,17747.10^{-5}$ \\
\hline $\boldsymbol{C}_{70}=-\boldsymbol{J}_{7}$ & $-3,523597646.10^{-7}$ & $-1,35777.10^{-5}$ \\
\hline $\boldsymbol{C}_{80}=-\boldsymbol{J}_{8}$ & $-2,047991918.10^{-7}$ & $-9,67487.10^{-6}$ \\
\hline $\boldsymbol{C}_{90}=-\boldsymbol{J}_{9}$ & $-1,206168362.10^{-7}$ & $1,54960.10^{-5}$ \\
\hline
\end{tabular}

\section{Potencial Gravitacional}

Consideremos um satélite artificial, exclusivamente sob a atração gravitacional de um corpo central, orbitando um corpo com distribuição não uniforme de massa. O potencial gravitacional considerado, expresso em termos de coeficientes harmônicos esféricos, é dado por (Morando, 1974):

$$
\begin{aligned}
& U=\frac{\mu}{r}\left(1-\sum_{n=2}^{\infty} \frac{J_{n} a_{e}^{n}}{r^{n}} P_{n} \operatorname{sen}(\phi)+\right. \\
& \left.\sum_{n=2}^{\infty} \sum_{m=1}^{n} \frac{J_{n, m} a_{e}^{n}}{r^{n}} P_{n, m} \operatorname{sen}(\phi) \cos m\left(\lambda-\lambda_{n, m}\right)\right)
\end{aligned}
$$

em que $n$ e $m$ são, respectivamente, o grau e a ordem do polinômio associado de Legendre, $\mu$ é a constante gravitacional, $a_{e}$ é o raio equatorial do corpo central considerado. $P_{n, m}$ são os polinômios associados de Legendre. $r$ é o raio vetor (distância entre o satélite e o centro de massa do corpo com distribuição não uniforme de massa). O ângulo $\phi$ é a latitude do satélite, $\lambda$ é a longitude; $J_{n}, J_{n, m}$ e $\lambda_{n, m}$ são características do corpo central. Tal potencial pode ser expresso em termos dos elementos orbitais do satélite (Morando, 1974). Os elementos orbitais métricos do satélite artificial: semieixo maior $(a)$, excentricidade $(e)$ e inclinação $(i)$ são introduzidos através de $r$. As variáveis angulares são introduzidas usando trigonometria esférica: longitude do nodo ascendente $(\Omega)$, argumento 
do pericentro $(\omega)$ e da anomalia verdadeira $(f)$ através da relação (Carvalho et. al., 2010):

$\operatorname{sen}(\phi)=\operatorname{sen}(i) \operatorname{sen}(f+\omega)$

\section{Variação secular dos elementos angulares}

Substituindo o potencial dado pela equação (1), nas equações planetárias de Lagrange, considerando apenas os termos seculares até a ordem de $\mathbf{J}_{2}$, temos as seguintes expressões para as variações dos elementos angulares (Morando, 1974):

$$
\begin{aligned}
& \Omega=n_{\Omega} t+\Omega_{0}= \\
& \left(-n J_{2}\left(\frac{a_{e}}{a}\right)^{2} \frac{1}{\left(1-e^{2}\right)^{2}} \frac{3}{2} \cos i\right) t+\Omega_{0}
\end{aligned}
$$

$$
\begin{aligned}
& \omega=n_{\omega} t+\omega_{0}= \\
& \left(n J_{2}\left(\frac{a_{e}}{a}\right)^{2} \frac{1}{\left(1-e^{2}\right)^{2}}\left(-\frac{3}{5}+\frac{15}{4} \cos ^{2} i\right)\right) t+\omega_{0}
\end{aligned}
$$

$$
n t+\left(3 n J_{2}\left(\frac{a_{e}}{a}\right)^{2}\left(-\frac{1}{4}+\frac{3}{4} \cos ^{2} i\right)\left(1-e^{2}\right)^{-\frac{3}{2}}\right) t+M_{0}
$$$$
M=n t+n_{M} t+M_{0}=
$$

Embora não haja perturbações seculares para elementos métricos $(a, e, i)$ quando se considera no potencial gravitacional apenas harmônicos zonais pares, as perturbações periódicas em tais elementos provocadas pelos outros harmônicos, aqui não consideradas, são importantes para missões envolvendo satélites lunares baixos.

As tabelas 2, 3 e 4 mostram a variação de $\omega, \Omega$ e $M\left(n_{\omega}, n_{\Omega}\right.$ e $\left.n_{M}\right)$, em graus por segundo, para alguns valores de $h=a-a_{e}$ e $i$ considerando os termos seculares até a ordem de $J_{2}$ (todos os

\begin{tabular}{|c|c|c|c|c|}
\hline & $\mathbf{h}=\mathbf{a}-\mathbf{a}_{e}$ & $\mathbf{n}_{\mathrm{w}}$ & $\mathbf{n}_{\Omega}$ & $\mathbf{n}_{\mathrm{M}}$ \\
\hline \multirow{3}{*}{ LUA } & $50 \mathrm{~km}$ & $0.0000226 \% \mathrm{~s}$ & $-0.0000132 \% / \mathrm{s}$ & $0.0000096 \% / \mathrm{s}$ \\
\hline & $100 \mathrm{~km}$ & $0.0000205 \% / \mathrm{s}$ & $-0.0000120 \% / \mathrm{s}$ & $0.0000087 \% / \mathrm{s}$ \\
\hline & $200 \mathrm{~km}$ & $0.0000170 \% / \mathrm{s}$ & $-0.0000100 \% / \mathrm{s}$ & $0.0000072 \% / \mathrm{s}$ \\
\hline \multirow{3}{*}{ TERRA } & $300 \mathrm{~km}$ & $0.0001448 \% / \mathrm{s}$ & $-0.0000850 \% / \mathrm{s}$ & $0.0000614 \% / \mathrm{s}$ \\
\hline & $350 \mathrm{~km}$ & $0.0001411 \% / \mathrm{s}$ & $-0.0000828 \circ / \mathrm{s}$ & $0.0000598 \% / s$ \\
\hline & $400 \mathrm{~km}$ & $0.0001375 \% / \mathrm{s}$ & $-0.0000807 \circ / \mathrm{s}$ & $0.0000583 \% / \mathrm{s}$ \\
\hline
\end{tabular}
resultados descritos nas tabelas e gráficos ao longo deste trabalho foram obtidos a partir dos

\begin{tabular}{|c|c|c|c|c|}
\hline & $h=a-a_{e}$ & $\mathbf{n}_{\omega}$ & $\mathbf{n}_{\Omega}$ & $\mathbf{n}_{\mathbf{M}}$ \\
\hline \multirow{3}{*}{ LUA } & $50 \mathrm{~km}$ & $0.0000034 \%$ & $-0.0000076^{\%} / \mathrm{s}$ & -0.0000019 \\
\hline & $100 \mathrm{~km}$ & $0.0000031 \%$ & $-0.0000069 \%$ & -0.0000017 \\
\hline & $200 \mathrm{~km}$ & $0.0000026 \%$ & $-0.0000058 \% \mathrm{~s}$ & -0.0000014 \\
\hline \multirow{3}{*}{ TERRA } & $300 \mathrm{~km}$ & $0.0000221 \%$ & $-0.0000491 \% \mathrm{~s}$ & -0.0000123 \\
\hline & $350 \mathrm{~km}$ & $0.0000215 \% \mathrm{~s}$ & $-0.0000478 \% \mathrm{~s}$ & -0.0000120 \\
\hline & $400 \mathrm{~km}$ & $0.0000210 \% \mathrm{~s}$ & $-0.0000466 \% \mathrm{~s}$ & -0.0000117 \\
\hline
\end{tabular}
softwares MAPLE e MATLAB):
Tabela 2. Valores de $\mathrm{n}_{\omega}, \mathrm{n}_{\Omega}$ e $\mathrm{n}_{\mathrm{M}}$ para $e=0.01 \mathrm{e} i=30^{\circ}$

\begin{tabular}{|c|c|c|c|c|}
\hline & $\mathbf{h}=\mathbf{a}-\mathbf{a}_{\mathrm{e}}$ & $\mathbf{n}_{\mathrm{w}}$ & $\mathbf{n}_{\Omega}$ & $\mathbf{n}_{\mathbf{M}}$ \\
\hline \multirow{3}{*}{ LUA } & $50 \mathrm{~km}$ & $-0.0000050 \%$ & $0.0000027 \%$ & $-0.0000070 \% \mathrm{~s}$ \\
\hline & $100 \mathrm{~km}$ & $-0.0000045^{\circ} / \mathrm{s}$ & $0.0000024 \%$ & $-0.0000063 \%$ \\
\hline & $200 \mathrm{~km}$ & $-0.0000037^{\circ} / \mathrm{s}$ & $0.0000020 \%$ & $-0.0000052 \%$ \\
\hline \multirow{3}{*}{ TERRA } & $300 \mathrm{~km}$ & $-0.0000319^{\prime} / \mathrm{s}$ & $0.0000170 \% \mathrm{~s}$ & $-0.0000446 \%$ \\
\hline & $350 \mathrm{~km}$ & $-0.0000311 \% \mathrm{~s}$ & $0.0000166 \% \mathrm{~s}$ & $-0.0000435 \% \mathrm{~s}$ \\
\hline & $400 \mathrm{~km}$ & $-0.0000303 \% \mathrm{~s}$ & $0.0000162 \% \mathrm{~s}$ & $-0.0000424 \% \mathrm{~s}$ \\
\hline
\end{tabular}

Tabela 3. Valores de $\mathrm{n}_{\omega}, \mathrm{n}_{\Omega}$ e $\mathrm{n}_{\mathrm{M}}$ para $e=0.01 \mathrm{e} i=60^{\circ}$

Tabela 4. Valores de $\mathrm{n}_{\omega}, \mathrm{n}_{\Omega}$, e $\mathrm{n}_{\mathrm{M}}$ para $e=0.01 \mathrm{e} i=100^{\circ}$.

Considerando novamente apenas termos seculares, e de longo período contendo coeficientes fatorados por $J_{2}$ e $J_{4}$ na equação (1), a expressão para a perturbação é dada por (Carvalho et al., 2012):

$$
\begin{aligned}
& U=-\frac{1}{512} \frac{1}{a^{2}}\left(n ^ { 2 } \left(72 J_{4} a_{e}^{4}-720 \cos ^{2}(i) J_{4} a_{e}^{4}\right.\right. \\
& +840 \cos ^{4}(i) J_{4} a_{e}^{4}+360 J_{4} a_{e}^{4} e^{2} \\
& +128 J_{2} a_{e}^{2} a^{2}+192 J_{2} a_{e}^{2} a^{2} e^{2} \\
& +945 J_{4} a_{e}^{4} e^{4}-384 \cos ^{2}(i) J_{2} a_{e}^{2} a^{2} \\
& -9450 \cos ^{2}(i) J_{4} a_{e}^{4} e^{4}-3600 \cos ^{2}(i) J_{4} a_{e}^{4} e^{2} \\
& +11025 \cos ^{4}(i) J_{4} a_{e}^{4} e^{4}+4200 \cos ^{4}(i) J_{4} a_{e}{ }^{4} e^{2} \\
& \left.\left.-576 \cos ^{2}(i) J_{2} a_{e}^{2} a^{2} e^{2}\right)\right)
\end{aligned}
$$

Substituindo (6) nas equações planetárias de Lagrange (3, 4, 5), temos as seguintes expressões para as variações dos elementos angulares: 
$\Omega=n_{\Omega} t+\Omega_{0}=\left(-\frac{1}{512} \frac{1}{a^{4} \sqrt{1-e^{2}} \operatorname{sen}(i)}\right.$

$\left(n\left(1440 \cos (i) J_{4} a_{e}{ }^{4} \operatorname{sen}(i)\right.\right.$

$-3360 \cos ^{3}(i) J_{4} a_{e}{ }^{4} \operatorname{sen}(i)$

$+768 \cos (i) J_{2} a_{e}{ }^{2} a^{2} \operatorname{sen}(i)$

$+18900 \cos (i) J_{4} a_{e}{ }^{4} e^{4} \operatorname{sen}(i)$

$+7200 \cos (i) J_{4} a_{e}{ }^{4} e^{2} \operatorname{sen}(i)$

$-44100 \cos ^{3}(i) J_{4} a_{e}{ }^{4} e^{4} \operatorname{sen}(i)$

$-16800 \cos ^{3}(i) J_{4} a_{e}{ }^{4} e^{2} \operatorname{sen}(i)$

$\left.\left.\left.+1152 \cos (i) J_{2} a_{e}^{2} a^{2} e^{2} \operatorname{sen}(i)\right)\right)\right) t+\Omega_{0}$

$\omega=n_{\omega} t+\omega_{0}=\left(\frac{1}{512} \frac{1}{a^{4} e}\left(\sqrt{1-e^{2}} n\right.\right.$

$\left(720 J_{4} a_{e}{ }^{4} e+384 J_{2} a_{e}{ }^{2} a^{2} e+3780 J_{4} a_{e}{ }^{4} e^{3}\right.$

$-37800 \cos ^{2}(i) J_{4} a_{e}{ }^{4} e^{3}-7200 \cos ^{2}(i) J_{4} a_{e}{ }^{4} e$

$+44100 \cos ^{4}(i) J_{4} a_{e}{ }^{4} e^{3}+8400 \cos ^{4}(i) J_{4} a_{e}{ }^{4} e$

$\left.-1152 \cos ^{2}(i) J_{2} a_{e}^{2} a^{2} e\right)$ )

$+\frac{1}{512} \frac{1}{a^{4} \sqrt{1-e^{2}} \operatorname{sen}(i)}(\cos (i) n$

$\left(1440 \cos (i) J_{4} a_{e}^{4} \operatorname{sen}(i)\right.$

$-3360 \cos (i)^{3} J_{4} a_{e}{ }^{4} \operatorname{sen}(i)$

$+768 \cos (i) J_{2} a_{e}{ }^{2} a^{2} \operatorname{sen}(i)$

$+18900 \cos (i) J_{4} a_{e}{ }^{4} e^{4} \operatorname{sen}(i)$

$+7200 \cos (i) J_{4} a_{e}^{4} e^{2} \operatorname{sen}(i)$

$-44100 \cos ^{3}(i) J_{4} a_{e}{ }^{4} e^{4} \operatorname{sen}(i)$

$-16800 \cos ^{3}(i) J_{4} a_{e}{ }^{4} e^{2} \operatorname{sen}(i)$

$\left.\left.\left.+1152 \cos (i) J_{2} a_{e}{ }^{2} a^{2} e^{2} \operatorname{sen}(i)\right)\right)\right) t+\omega_{0}$

$M=n t+n_{M} t+M_{0}=n t+\left(n-\frac{1}{n a}\left(2\left(-\frac{1}{512} \frac{1}{a^{2}}\left(n^{2}\left(256 J_{2} a_{e}^{2} a\right.\right.\right.\right.\right.$

$\left.\left.+384 J_{2} a_{e}^{2} a e^{2}-768 \cos ^{2}(i) J_{2} a_{e}^{2} a-1152 \cos ^{2}(i) J_{2} a_{e}^{2} a e^{2}\right)\right)$

$+\frac{1}{256} \frac{1}{a^{3}}\left(n^{2}\left(72 J_{4} a_{e}{ }^{4}-720 \cos ^{2}(i) J_{4} a_{e}{ }^{4}+840 \cos ^{4}(i) J_{4} a_{e}{ }^{4}\right.\right.$

$+360 J_{4} a_{e}{ }^{4} e^{2}+128 J_{2} a_{e}{ }^{2} a^{2}+192 J_{2} a_{e}{ }^{2} a^{2} e^{2}+945 J_{4} a_{e}{ }^{4} e^{4}$

$-384 \cos ^{2}(i) J_{2} a_{e}{ }^{2} a^{2}-9450 \cos ^{2}(i) J_{4} a_{e}{ }^{4} e^{4}$

$-3600 \cos ^{2}(i) J_{4} a_{e}{ }^{4} e^{2}+11025 \cos ^{4}(i) J_{4} a_{e}{ }^{4} e^{4}$

$\left.\left.\left.\left.+4200 \cos ^{4}(i) J_{4} a_{e}^{4} e^{2}-576 \cos ^{2}(i) J_{2} a_{e}^{2} a^{2} e^{2}\right)\right)\right)\right)$

$+\frac{1}{512} \frac{1}{a^{4} e}\left(\left(1-e^{2}\right) n\left(720 J_{4} a_{e}{ }^{4} e+384 J_{2} a_{e}{ }^{2} a^{2} e\right.\right.$

$+3780 J_{4} a_{e}{ }^{4} e^{3}-37800 \cos ^{2}(i) J_{4} a_{e}^{4} e^{3}$

$-7200 \cos (i)^{2} J_{4} a_{e}{ }^{4} e+44100 \cos ^{4}(i) J_{4} a_{e}{ }^{4} e^{3}$

$\left.\left.\left.+8400 \cos ^{4}(i) J_{4} a_{e}{ }^{4} e-1152 \cos ^{2}(i) J_{2} a_{e}{ }^{2} a^{2} e\right)\right)\right) t+M_{0}$

As tabelas 5, 6 e 7 mostram a variação de $\omega, \Omega$ e $M\left(n_{\omega}, n_{\Omega}\right.$ e $\left.n_{M}\right)$ em graus por segundo para alguns valores de $h=a-a_{e}$ e $i$ considerando os termos seculares até a ordem de $J_{4}$.

Uma expressão para o potencial com termos de curto e longo período, envolvendo harmônicos de maior grau e ordem pode ser encontrada em (Carvalho et al., 2012).
Tabela 5. Valores de $\mathrm{n}_{\omega}, \mathrm{n}_{\Omega}$, e $\mathrm{n}_{\mathrm{M}}$ para $e=0.01 \mathrm{e} i=30^{\circ}$

\begin{tabular}{|c|c|c|c|c|}
\cline { 2 - 5 } \multicolumn{1}{c|}{} & h=a-ae & $\mathbf{n}_{\omega}$ & $\mathbf{n}_{\Omega}$ & $\mathbf{n}_{\mathbf{M}}$ \\
\hline \multirow{3}{*}{ LUA } & $50 \mathrm{~km}$ & $-0.0000026 \% / \mathrm{s}$ & $-0.0000141 \% / \mathrm{s}$ & $-0.0000097 \% / \mathrm{s}$ \\
& $100 \mathrm{~km}$ & $-0.0000023 \% / \mathrm{s}$ & $-0.0000127 \% / \mathrm{s}$ & $-0.0000088 \% / \mathrm{s}$ \\
& $200 \mathrm{~km}$ & $-0.0000019 \% / \mathrm{s}$ & $-0.0000105 \% / \mathrm{s}$ & $-0.0000073 \% / \mathrm{s}$ \\
\hline \multirow{3}{*}{ TERRA } & $300 \mathrm{~km}$ & $-0.0000124 \% / \mathrm{s}$ & $-0.0000852 \% / \mathrm{s}$ & $-0.000614 \% / \mathrm{s}$ \\
& $350 \mathrm{~km}$ & $-0.0000121 \% / \mathrm{s}$ & $-0.0000830 \% / \mathrm{s}$ & $-0.0000599 \% / \mathrm{s}$ \\
& $400 \mathrm{~km}$ & $-0.0000118 \% / \mathrm{s}$ & $-0.0000809 \% / \mathrm{s}$ & $-0.0000583 \% / \mathrm{s}$ \\
\hline
\end{tabular}

Tabela 6. Valores de $\mathrm{n}_{\omega}, \mathrm{n}_{\Omega}$, e $\mathrm{n}_{\mathrm{M}}$ para $e=0.01 \mathrm{e} i=60^{\circ}$.

\begin{tabular}{|c|c|c|c|c|}
\hline & $h=a-a e$ & $\mathbf{n}_{\mathrm{e}}$ & $\mathbf{n}_{\Omega}$ & $\mathbf{n}_{\mathbf{M}}$ \\
\hline \multirow{3}{*}{ LUA } & $50 \mathrm{~km}$ & $-0.0000061 \% / \mathrm{s}$ & $-0.0000074 \% / s$ & $0.0000032 \% / \mathrm{s}$ \\
\hline & $100 \mathrm{~km}$ & $-0.0000055 \% / \mathrm{s}$ & $-0.0000067 \% / \mathrm{s}$ & $0.0000028 \% / \mathrm{s}$ \\
\hline & $200 \mathrm{~km}$ & $-0.0000046 \% / \mathrm{s}$ & $-0.0000056 \% / \mathrm{s}$ & $0.0000022 \% / \mathrm{s}$ \\
\hline \multirow{3}{*}{ TERRA } & $300 \mathrm{~km}$ & $-0.0000369 \% / \mathrm{s}$ & $-0.0000490 \% / \mathrm{s}$ & $0.0000135 \% / \mathrm{s}$ \\
\hline & $350 \mathrm{~km}$ & $-0.0000359 \% / \mathrm{s}$ & $-0.0000478 \circ / \mathrm{s}$ & $0.0000131 \% / \mathrm{s}$ \\
\hline & $400 \mathrm{~km}$ & $-0.0000350 \% / \mathrm{s}$ & $-0.0000466 \% / \mathrm{s}$ & $0.0000127 \% / \mathrm{s}$ \\
\hline
\end{tabular}

Tabela 7. Valores de $\mathrm{n}_{\omega}, \mathrm{n}_{\Omega}$, e $\mathrm{n}_{\mathrm{M}}$ para $e=0.01 \mathrm{e} i=100^{\circ}$.

\begin{tabular}{|c|c|c|c|c|}
\cline { 2 - 5 } \multicolumn{1}{c|}{} & $\mathbf{h}=\mathbf{a}-\mathbf{a}_{\mathrm{e}}$ & $\mathbf{n}_{\mathrm{w}}$ & $\mathbf{n}_{\Omega}$ & $\mathbf{n}_{\mathrm{M}}$ \\
\hline \multirow{3}{*}{ LUA } & $50 \mathrm{~km}$ & $-0.0000069 \% \mathrm{~s}$ & $0.0000024 \% \mathrm{~s}$ & $0.0000058 \% / \mathrm{s}$ \\
& $100 \mathrm{~km}$ & $-0.0000063 \% \mathrm{~s}$ & $0.0000022 \% / \mathrm{s}$ & $0.0000053 \% / \mathrm{s}$ \\
& $200 \mathrm{~km}$ & $-0.0000053 \% \mathrm{~s}$ & $0.0000019 \% / \mathrm{s}$ & $0.0000046 \% / \mathrm{s}$ \\
\hline \multirow{3}{*}{ TERRA } & $300 \mathrm{~km}$ & $-0.0000475 \% / \mathrm{s}$ & $-0.0000170 \% / \mathrm{s}$ & $0.0000435 \% / \mathrm{s}$ \\
& $350 \mathrm{~km}$ & $-0.0000463 \%$ & $0.0000166 \% \mathrm{~s}$ & $0.0000424 \%$ \\
& $400 \mathrm{~km}$ & $-0.0000451 \%$ & $0.0000161 \% / \mathrm{s}$ & $0.0000414 \%$ \\
\hline
\end{tabular}

\section{Inclinação Crítica e Órbitas Heliossíncronas}

Órbitas com inclinação crítica e órbitas heliossíncronas são comumente utilizadas por satélites com finalidade de sensoriamento remoto. A influencia dos harmônicos de alta ordem e grau para inclinações críticas e órbitas heliossíncronas de satélites lunares também é significativa, de fato, considerando-se $\mathrm{J}_{2}$ e $\mathrm{C}_{22}$ temos as seguintes expressões para inclinação crítica e inclinações de órbitas heliossíncronas (Carvalho et al., 2009):

$$
\cos ^{2}(i)=\frac{-J_{2} a_{e}{ }^{2}+6 C_{22} a_{e}{ }^{2} \cos (2 \Omega)}{5\left(-J_{2} a_{e}{ }^{2}+2 C_{22} a_{e}{ }^{2} \cos (2 \Omega)\right)}
$$


$i_{s}=\pi-$

$\arccos \left(\frac{2,5462.10^{-7} a^{2} \sqrt{1-e^{2}}}{n\left(2 \varepsilon+6 \delta \cos (2 \Omega) e^{2}+4 \delta \cos (2 \Omega)+3 \varepsilon e^{2}\right)}\right)$

em que:

$$
\begin{aligned}
& \varepsilon=J_{2} a_{e}{ }^{2} \\
& \delta=-C_{22} a_{e}{ }^{2}
\end{aligned}
$$

Assim, vemos que para um satélite lunar em uma órbita congelada a inclinação crítica seria $63,43^{\circ}$ se considerassemos apenas a influência do $J_{2}$. Entretanto considerando-se a influência do harmônico $C_{22}$, vemos pela equação (10) que a inclinação depende de $\Omega$. Na Figura 1 observa-se que nesse caso a inclinação crítica pode variar entre $58,77^{\circ}$ e $72,62^{\circ}$. Para satélites artificiais terrestres a variação é muito pequena quando se considera o $C_{22}$.

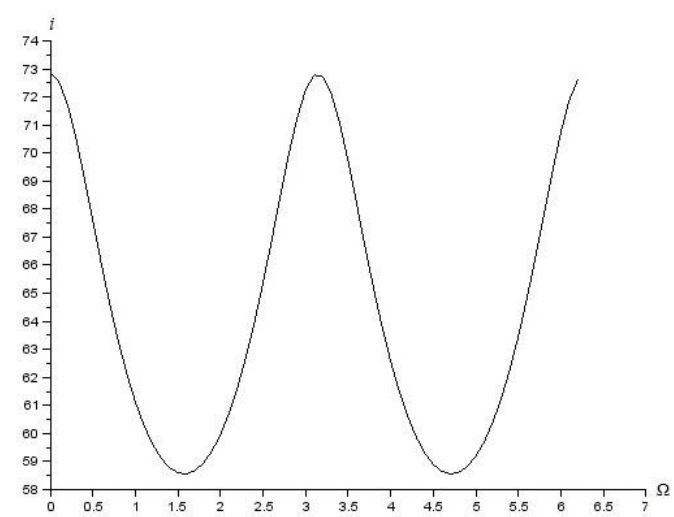

Figura 1. Variação da inclinação crítica em relação à longitude do nodo ascendente em que $i$ está em graus e $\Omega$ está em radianos.

Para satélites lunares também as órbitas heliossíncronas são marcadamente influenciadas pelo harmônico $C_{22}$.

A Figura 2 ilustra a variação da inclinação de órbitas heliossíncronas para dois satélites lunares baixos $(a=1838 \mathrm{~km})$, um em órbita circular e outro com excentricidade de 0,038 .

Também, para órbitas heliossíncronas de satélites terrestres, devido à ordem $C_{22}$ para a Terra, a influência na variação da inclinação é não considerável.

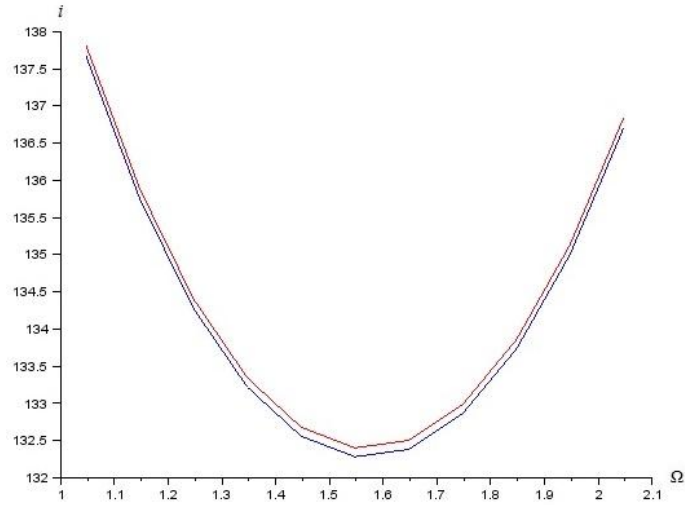

Figura 2. Variação da inclinação de duas órbitas heliossíncronas em relação à longitude do nodo ascendente em que as curvas vermelha e azul possuem excentricidade $e=0, e=0.038$ respectivamente, $a=1838 \mathrm{~km}, i$ está em graus e $\Omega$ está em radianos.

\section{Conclusão}

Verificamos que a influência de considerarmos mais harmônicos no cálculo da variação dos elementos keplerianos angulares é significativamente maior para o caso de satélites lunares do que para satélites artificiais terrestres, mesmo para satélites altos.

\section{Agradecimentos}

Os autores agradecem a FAPESP, CAPES, CNPq e ao Fundo de Auxílio aos Docentes e Alunos da Universidade Federal de São Paulo (FADA) pelo apoio financeiro.

\section{Referências Bibliográficas}

Carvalho, J. P. S., Vilhena de Moraes, R., Prado, A. F. B. A. Planetary Satellite Orbiters: Applications for the Moon. Mathematical Problems in Engineering, DOI: $10.1155 / 2011 / 187478$

Carvalho, J. P. S., Vilhena de Moraes, R., Prado, A. F. B. A. Some orbital characteristics of lunar artificial satellites. Celestial Mechanics \& Dynamical Astronomy, vol. 108, $\mathrm{n}^{\circ}$ 4, pp. 371-388, 2010. DOI: 10.1007/s10569-010-9310-6

Carvalho, J. P. S., Vilhena de Moraes, R., Prado, A. F. B. A. Nonsphericity of the Moon and Near Sun-Synchronous Polar Lunar Orbits. Mathematical Problems in Engineering, doi: 10.1155/2009/740460, 2009. DOI: $10.1155 / 2009 / 740460$

Chen, J. Y., Ning, J. S., Zhang, C. Y., Lou, J. 
On the determination of lunar gravity field in the Chinese first lunar prospector mission. Chinese Journal of Geophysics, vol. $48, \mathrm{n}^{\circ} 2$, pp. 275-281, 2005.

Leimoine, F., Kenyon, S., Factor, J., Trimmer, R., Pavlis, N., Chinn, D., Cox, C., Klosko, S., Luthcke, S., Torrence, M., Wang, Y., Williamson, E., Rapp, R., Olson, T. The development of the joint NASA - GSFC and the NIMA Geopotential Model EGM 96. NASA/TP -206861. 1998.

Morando, M. B. Mouvement d'un satellite artificiel de la Terre. Gordon \& Breach, Paris, 1974. 